\title{
A review of complications of maxillary sinus augmentation and available treatment methods
}

\author{
Joongmin $\mathrm{Kim}^{1,2}$, Hyonseok Jang ${ }^{2}$ \\ ${ }^{1}$ Department of Oral and Maxillofacial Surgery, Private Clinic, Seoul, \\ ${ }^{2}$ Department of Oral and Maxillofacial Surgery, Korea University Ansan Hospital, Ansan, Korea
}

\begin{abstract}
J Korean Assoc Oral Maxillofac Surg 2019;45:220-224)
Maxillary sinus grafting is a dependable procedure that has been in use for a long time. However, clinical complications often arise. To prevent complications of maxillary sinus grafting, it is necessary to know the contra-indications, both for general implantation and for maxillary bone grafting. In addition, presence of various complications requires careful consideration of treatment method; therefore, dentists should be familiar with the treatment protocols. Complications can be divided into postoperative, immediate postoperative, and delayed postoperative complications. Particularly for the outpatient, it is necessary to quickly distinguish between treatable cases and cases for which transfer is required. The purpose of this review is to discuss the contra-indications, complications, and treatment options for complications of maxillary sinus graft.
\end{abstract}

Key words: Maxillary sinus, Contraindication, Complication

[paper submitted 2019. 6. 10 / revised 2019. 6. 19 / accepted 2019. 6. 19]

\section{Introduction}

Dr. Hilt Tatum proposed a modified Caldwell-Luc operation as a maxillary sinus graft in the 1970 s, and it has been recognized as a procedure with high predictability to date ${ }^{1}$. However, as in all surgical procedures, various complications may arise after maxillary sinus graft ${ }^{2}$. In this review, we aimed to investigate the types of complications associated with maxillary sinus graft, methods for prevention of said complications, and existing treatment options available in dental clinics.

A complication is a problem or difficulty that makes a situation harder to deal with, and multiple complications tend to occur at the same time in medicine ${ }^{3}$. In order to prevent complications in the present study, it was first necessary to

\section{Joongmin Kim}

Department of Oral and Maxillofacial Surgery, Private Clinic, Suite \#214, Daebang B/D, 10-4, Yeouidaebang-ro 24-gil, Dongjak-gu, Seoul 07056, Korea

TEL: +82-2-821-0047 FAX: +82-2-6455-3771

E-mail: kjm-0730@daum.net

ORCID: https://orcid.org/0000-0002-9395-2232

(c) This is an open-access article distributed under the terms of the Creative Commons Attribution Non-Commercial License (http://creativecommons.org/ licenses/by-nc/4.0/), which permits unrestricted non-commercial use, distribution, and reproduction in any medium, provided the original work is properly cited.

Copyright (C) 2019 The Korean Association of Oral and Maxillofacial Surgeons. All rights reserved. correctly identify and differentiate the indications and contraindications for maxillary sinus surgery.

\section{Contraindications}

The contraindications of maxillary sinus augmentation can be divided into general implant contraindications, absolute contraindications, and relative contraindications. Needless to say, maxillary sinus graft is not an appropriate treatment option for patients with general implant contraindications. Absolute contraindications include severe or uncontrollable general disease, large-dose radiation therapy on the maxilla, mental disorders, sepsis, heavy smoking, and severe alcoholism or drug abuse. On the other hand, the factors constituting relative contraindications are local hard or soft tissue lesions such as sinus infection, acute infection (dental origin), pathologic condition of the sinus (polyp, cyst, and tumor), and sinusitis including allergic rhinitis; history of sinus surgery; low-dose radiation therapy on the maxilla, habitual use of drugs, alcohol, and tobacco; mouth opening limitations; malocclusion; and severe bruxism ${ }^{4}$.

In all dental procedures including implant placement, careful case selection is likely to lead to a successful outcome. For example, if sinusitis, a maxillary cyst, a maxillary tumor, or a root resting in the sinus is detected in the maxillary sinus, 
it is necessary to delay all surgical procedures and first treat the lesions or infections that may affect surgical outcomes.

The mucous retention cyst (MRC), a pseudocyst without epithelial cell lining accumulating liquid under the sinus, is usually observed as a dome or ball shape. In the case of a MRC, performing simultaneous or delayed maxillary sinus graft and implant placement under local anesthesia can be a first treat option in the dental clinic. When MRC is observed on panoramic radiography or cone-beam computed tomography $(\mathrm{CBCT})$ images, it is necessary to first elevate the flap and form a bone window at a preferred location according to the general principle ${ }^{5}$. A $10 \mathrm{~mL}$ syringe and a 21 -gauge needle is used to aspirate the fluid from the inside of the cyst and, if necessary, the inside should be irrigated with an saline solution including antibiotics. Then, according to the conventional procedure, the membrane of the cyst is pushed up so that the membrane of the maxillary sinus is elevated and bone grafting is performed, followed by implant placement. If the size of the cyst is smaller than $10 \mathrm{~mm}$, it is difficult to regard it as a contraindication because it can be treated by suction during surgery. However, if the size of the cyst is larger than $10 \mathrm{~mm}$, a staged approach should be considered to wait for aspiration and to perform maxillary sinus graft after delaying it for some time after aspiration. The ostium or osteomaltal complex (OMC) may be blocked when aspiration is not properly performed.

\section{Complications}

Complications associated with maxillary sinus augmentation can be categorized into intraoperative complications, acute postoperative complications, and chronic postoperative complications. The most frequent complications of maxillary sinus graft were perforation or tear $(60 \%)$, infection $(21 \%)$, bleeding $(9 \%)$, migration, benign paroxysmal positional vertigo (BPPV) in descending order ${ }^{6}$.

\section{Intraoperative complications}

The known intraoperative complications of maxillary sinus graft include tearing of the Schneiderian membrane, antral or nasal penetration, bleeding, damage or injury of adjacent teeth, fracture, fenestration, dehiscence or perforation of alveolar bone, insufficient primary stability, improper position or angulation of the fixture, obstruction of the OMC, and swallowing of instruments. Tearing of the Schneiderian membrane generally occurs due to preexisting perforation, exist- ing or previous pathologic condition, tearing during scoring of the lateral window, or elevation from bony walls ${ }^{7}$. The rupture of the Schneiderian membrane is the most common complication of maxillary sinus graft and it is reported to occur in $10 \%$ to $34 \%$ of patients. The incidence rate is higher in smokers than nonsmokers because the maxillary sinus membrane becomes thinner in cigarette smoke.

If the perforation of the maxillary sinus membrane is smaller than $5 \mathrm{~mm}$, the survival rate of implants has been reported to be $97.14 \%$, which is not significantly different from the survival rate of normal implants. However, since the survival rate is decreased to $91.89 \%$ in cases of perforations with a size of 5 to $10 \mathrm{~mm}$ and to $74.14 \%$ in cases of perforations larger than $10 \mathrm{~mm}$, special care should be taken during surgery to avoid tearing the membrane ${ }^{8}$. In addition, since it has been reported that, when there is a perforation of the maxillary sinus membrane, simultaneous implant placement leads to a survival rate of $90.81 \%$, there is a need to consider a staged approach ${ }^{9}$.

Previously, we repaired large punches using Loma Linda pouch technique. To do this, a large membrane is placed inside the maxillary sinus to create a pouch and implant the bone graft material in the pouch. However, since there were reported cases wherein the membrane prevented osteocytes from migrating from the periosteum, leading to bone graft failures, it is generally not used any longer.

Next, we will discuss factors related to bleeding and bleeding control techniques. The infraorbital artery and posterior superior alveolar artery run in and out of the lateral wall of the maxillary sinus, forming an extraosseous anastomosis and intraosseous anastomosis. As for the intraosseous branch, it has been reported that straight-type branches compose $75 \%$ of total cases, while U-shaped ones make up $25 \%$. If only a part of the intraosseous branch is observed on the CBCT, it is possible to predict the routes of blood vessels based on the above data. In the case of the intraosseous branch, the distance from the cementoenamel juntion (CEJ) of the posterior maxilla to the vessel varies from a minimum of $15.2 \mathrm{~mm}$ to a maximum of $34.6 \mathrm{~mm}$, with an average of 21.1 to 26.9 $\mathrm{mm}$. Additionally, the distance from the inferior border of the maxillary sinus to the vessel ranges from a minimum of $2.5 \mathrm{~mm}$ to a maximum of $19.4 \mathrm{~mm}$, with an average of 9.4 to $10.3 \mathrm{~mm}$. This means that, if a small window is formed and positioned as low as possible, there is a reduced chance of encountering the intraosseous branch. Recently, a variety of sinus lateral approach kits (e.g., SLA kit [Neobiotech, Seoul, Korea] and DASK [Dentium, Seoul, Korea]) have been de- 
veloped and are very helpful tools for surgery.

If excessive bleeding occurs during surgery, various techniques are used to control the bleeding. The first treatment for bleeding is to lift the patient's head position and pressing. When there is no pulse rhythm in the intra-osseous branch, bleeding can be controlled with gauze packing alone in most cases. If bleeding is not controlled only by pressing, the method of crushing the bone at the bleeding point using a bone rongeur or hemostat may be employed. Another method for bleeding control is to use a electrocoagulator or laser if available $^{10}$. However, if such instruments are not available, a high-speed handpiece can be installed with a diamond bur and applied to the bleeding point without irrigation to control. Alternatively, a periosteal elevator or other surgical instruments can be heated with an alcohol lamp and applied on the bleeding point. Commercial hemostatics such as Surgicel (Johnson \& Johnson, New Brunswick, NJ, USA) or bone wax may also be helpful.

\section{Acute postoperative complications}

Acute postoperative complications include pain, swelling, edema, infection of the surgical site and sinus, sinusitis, bone resorption, bleeding, oral and nasal ecchymosis and hematoma (especially hemosinus), emphysema, wound dehiscence, incisional breakdown, the loss of the graft, dislocation, migration or loss of the fixture, oroantral fistula, BPPV, and temporary or permanent palatal numbness.

The typical symptoms of sinusitis include foul odor, headache (migraine), midfacial discomfort while running, pressure with head position change, nasal obstruction, and tenderness. The common symptoms of sinus infection are facial pain, swelling, tenderness, pus discharge, and purulent nasal exudate.

For the treatment of sinus infection, antibiotics should be administered for at least two weeks together with a glucocorticoid, decongestant, and analgesic. In addition, cryotherapy can be used as needed. The drug of choice for sinus infection is Augmentin. Among analgesics, Tylenol (Johnson \& Johnson) is useful because it has an antitussive effect.

Now, we will describe an approach to be used in a hospital when the symptoms of the sinus are not controlled by medication or when symptoms develop suddenly and rapidly and the patient feels very uncomfortable. First, a small incision is made on the easily accessible side wall area of the maxillary sinus using a blade. The flap is elevated to a small extent, and a No. 4 carbide round bur is used to pierce the wall of the maxillary sinus. The irritants inside the maxillary sinus should be aspirated using a syringe with a needle as large as 18-gauge. After asking patients to hold their breaths, saline is injected into the maxillary sinus, and aspiration is performed again. If saline comes out of the patient's nose or mouth, the patient should be instructed to sit in an upright position and then spit it out. An saline solution with antibiotics should be made and injected into the maxillary sinus. Suturing is not necessary and the same procedure should be repeated by requiring the patient to visit the clinic every day until symptoms are relieved. If the symptoms are not relieved by this method, the patient should be referred to the department of ear, nose and throat so that the patient can undergo functional endoscopic sinus surgery (FESS) ${ }^{11}$.

The migration of a fixture into the maxillary sinus poses a perplexing problem for clinicians. This problem is dealt with using the same approach as that employed in the CaldwellLuc operation ${ }^{12}$. Here, a hole through which the fixture can come out is formed in the anterior wall of the maxillary sinus ${ }^{7}$. Since the fixture inside the maxillary sinus is constantly changing its position, it is often difficult to insert a device and hold it with said device. In many cases, it can be removed it by injecting saline into the maxillary sinus after asking the patient to hold their breath for a moment and then applying a metal suction tip with a small end on the hole.

In order to prevent migration of a fixture into the maxillary sinus, it is important to carry out initial fixation properly by correctly judging the bone quality at the stage of initial drilling and using under-size drilling if the bone quality is poor. In this respect, taper-type implants have advantages over straight-type implants, and greater thread depths are more advantageous.

If the technique of improving the bone quality or elevating the inferior border of the maxillary sinus by malleting with an osteotome is used, BPPV may occur. This disorder occurs when otoliths (ear rocks) in the utricular macula are detached by the impact of malleting and move each time the head position of the patient is changed, causing dizziness. If the use of an osteotome is necessary, the patient must be informed about the risk of BPPV before surgery. BPPV frequently occurs in people aged 50 to 69 years, and the incidence of BPPV increases with age. It has been reported to occur in about four out of 146 cases $(2.74 \%)$. When BPPV occurs, the symptoms are improved by the Epley maneuver. The Epley maneuver is a method of returning displaced otoliths to their original position by changing the position and head direction of the patient. 


\section{Chronic postoperative complications}

Finally, chronic postoperative complications include infection, sinusitis, implant periapical lesion, and postoperative maxillary cyst.

Although implant periapical lesions rarely occur in the maxilla, they occasionally occur in clinical cases when excessive heat is generated during drilling. If the bone is judged to be hard, it is good to have a long time interval (at least one minute) between drilling processes. It is also helpful to use chilled saline rather than the usual saline solution at room temperature. In theory, an internal irrigation drill has the advantage of preventing a temperature rise inside the bone, but it has the disadvantage of that, since the tube inside the drill is very narrow, it is difficult to disinfect and clean. Another drawback is that, when irrigation of the handpiece is used, it is difficult to perform irrigation at an exact position in implant procedures where drills with various lengths are used. Thus, it is important to perform irrigation at a precise position using a syringe. If irrigation is performed by inserting a syringe needle tip into the inside part where osteotomy was performed during drilling, the temperature inside the bone will be lowered without fail and bone chips generated during drilling can be completely removed.

Numbness often occurs in the mandible for various causes. However, even in the maxilla, idiopathic neuralgia may occur postoperatively, albeit very rarely. Idiopathic neuralgia is thought to be a central cause of pain, not a peripheral one, and often presents even when there is no nerve damage. Idiopathic neuralgia is subdivided into five categories: anaesthesiadolorosa, central poststroke pain, facial pain attributed to multiple sclerosis, persistent idiopathic facial pain (PIFP), and burning mouth syndrome. If the symptoms develop after oral surgery, PIFP is most likely to be the cause. With respect to PIFP, pain is not related to sensory loss and radiographic singularities cannot be detected. In addition, in the case of PIFP, pain is unilateral or limited to a specific area and shows a pattern of shooting pain. PIFP is associated with surgery or facial damage and, although the exact causes of PIFP are not known yet, a neuropathic pathology can be suspected. A tricyclic antidepressant (amitriptyline $25-100 \mathrm{mg}$ /day) or an anticonvulsant such as clonazepam is used for treatment. The symptoms of PIFP need to be differentiated from those of Bell's palsy.

The main functions of the maxillary sinus are the reduction of head weight, control of temperature and humidity of air, resonance of sound, and discharge of secretions. Maxillary si- nus graft leads to the reduction of the volume of the maxillary sinus. In this regard, it has been reported that maxillary sinus graft may result in changes in resonance due to the volume change of the maxillary sinus ${ }^{13,14}$. Therefore, if the patient belongs to a certain occupational group such as singers, actors, or voice actors, it is important to inform the patient about the possibility of changes in speech before surgery.

\section{Conclusion}

Among dental disputes, disputes about dental implants make up the second largest percentage, following dispute cases about nerve damage. In particular, maxillary sinus augmentation is a sensitive technique that involves a high risk of complications. Therefore, radiographic evaluation such as panorama, water's view, and CBCT should be performed to check for lesions in the maxillary sinus before surgery ${ }^{15}$. Because maxillary sinus augmentation may lead to various complications such as maxillary sinus perforation and maxillary sinusitis, sufficient explanations about possible complications should be given to all patients before surgery. Especially, elderly patients or patients with a systemic disease should be informed in detail about the higher risk for infections and the severity of the infection because of their lower resistance to infections, so that these individuals can understand the risks involved.

\section{ORCID}

Joongmin Kim, https://orcid.org/0000-0002-9395-2232

Hyonseok Jang, https://orcid.org/0000-0002-8493-4657

\section{Authors' Contributions}

J.K. participated in data collection and wrote the manuscript. H.J. participated in the study design and coordination and helped to draft the manuscript. All authors read and approved the final manuscript.

\section{Conflict of Interest}

No potential conflict of interest relevant to this article was reported. 


\section{References}

1. Tatum H Jr. Maxillary and sinus implant reconstructions. Dent Clin North Am 1986;30:207-29.

2. Bathla SC, Fry RR, Majumdar K. Maxillary sinus augmentation. J Indian Soc Periodontol 2018;22:468-73.

3. Jeong KI, Kim SG, Oh JS, You JS. Implants displaced into the maxillary sinus: a systematic review. Implant Dent 2016;25:54751.

4. Schwartz-Arad D, Herzberg R, Dolev E. The prevalence of surgical complications of the sinus graft procedure and their impact on implant survival. J Periodontol 2004;75:511-6.

5. Jang HY, Kim HC, Lee SC, Lee JY. Choice of graft material in relation to maxillary sinus width in internal sinus floor augmentation. J Oral Maxillofac Surg 2010;68:1859-68.

6. Barone A, Santini S, Sbordone L, Crespi R, Covani U. A clinical study of the outcomes and complications associated with maxillary sinus augmentation. Int J Oral Maxillofac Implants 2006;21:81-5.

7. Kim JH, Kim UC, Lee JY, Kim HC, Kim SN. A clinical \& radiologic study of bone remodeling effects using rhBMP-2 for maxillary sinus graft. J Dent Implant Res 2016;35:46-52.

8. Kim YK, Kim JA. Evaluation of factors which affect the prognosis of sinus bone graft and implant placement. J Dent Implant Res 2012;31:31-7.

9. Hernández-Alfaro F, Torradeflot MM, Marti C. Prevalence and management of Schneiderian membrane perforations during sinuslift procedures. Clin Oral Implants Res 2008;19:91-8.
10. Ella B, Sédarat C, Noble Rda C, Normand E, Lauverjat Y, Siberchicot $\mathrm{F}$, et al. Vascular connections of the lateral wall of the sinus: surgical effect in sinus augmentation. Int J Oral Maxillofac Implants 2008;23:1047-52.

11. Pignataro L, Mantovani M, Torretta S, Felisati G, Sambataro G. ENT assessment in the integrated management of candidate for (maxillary) sinus lift. Acta Otorhinolaryngol Ital 2008;28:110-9.

12. Low WK. Complications of the Caldwell-Luc operation and how to avoid them. Aust N Z J Surg 1995;65:582-4.

13. Tepper G, Haas R, Schneider B, Watzak G, Mailath G, Jovanovic SA, et al. Effects of sinus lifting on voice quality. A prospective study and risk assessment. Clin Oral Implants Res 2003;14:767-74.

14. Ungor C, Saridoğan C, Yilmaz M, Tosun E, Senel FC, Icten O, et al. An acoustical analysis of the effects of maxillary sinus augmentation on voice quality. Oral Surg Oral Med Oral Pathol Oral Radiol 2013;115:175-84.

15. Cha JY, Mah J, Sinclair P. Incidental findings in the maxillofacial area with 3-dimensional cone-beam imaging. Am J Orthod Dentofacial Orthop 2007;132:7-14.

How to cite this article: Kim J, Jang H. A review of complications of maxillary sinus augmentation and available treatment methods.

J Korean Assoc Oral Maxillofac Surg 2019;45:220-224. https://doi. org/10.5125/jkaoms.2019.45.4.220 